\title{
Comparativo entre as bases de dados PubMed, SciELO e Google Acadêmico com o foco na temática Educação Médica
}

\author{
Comparative between the PubMed, SciELO and Google Scholar \\ databases with the focus on thematic Medical Education.
}

\author{
${ }^{1}$ Lucas Rebelo Silva Puccini \\ ${ }^{1}$ Mara Gonçalves Pinto Giffoni \\ ${ }^{1}$ Leoni Ferreira da Silva \\ ${ }^{2}$ Claudia Yamada Utagawa
}

1 Discente do Curso de Medicina do Centro Universitário de Volta Redonda - UniFOA.
2 Docente do Curso de Medicina do Centro Universitário de Volta Redonda - UniFOA.

\section{RESUMO}

Introdução: A comunidade biomédica latino-americana tem o PubMed e o SciELO como as melhores bases de dados para pesquisa de artigos científicos, entretanto, outras áreas de conhecimento têm utilizado outras fontes de busca, sendo a principal, o Google Acadêmico (GA). Objetivos: Verificar se o GA pode ser uma ferramenta de busca útil no resgate de artigos científicos referenciados na temática em educação médica, comparados ao PubMed e ao Scielo. Metodologia: Trata-se de um estudo descritivo e documental com análise qualiquantitativa sobre os artigos científicos publicados no GA, PubMed e SciELO. Em cada sítio de busca foram utilizadas as seguintes palavraschave em inglês para a recuperação de dados: "medical curriculum" and "ethics". Resultados: Após a aplicação da técnica de mineração de dados nas plataformas de busca, foram obtidos 434 artigos científicos no GA, sendo 88 artigos disponíveis no site dos periódicos; 201 , no site das bases de dados; 143, em outros sites e, apenas dois, em blogs. No PubMed, foram obtidos 10 artigos científicos, sendo 8 disponíveis no site da base de dados e dois em sites de periódicos. Por fim, no SciELO, foi encontrado apenas um artigo, o qual estava disponível em sua própria base de dados. Qualitativamente, foram encontrados 382 artigos científicos pertinentes no GA, no PubMed e SciELO. Conclusão: O GA mostrou ser mais eficiente, tanto qualitativamente quanto quantitativamente, em resgatar artigos científicos sobre o tema escolhido em Educação Médica.

\section{Palavras-chave}

Bases de dados bibliográficas; educação médica; PubMed.

\begin{abstract}
Introduction: The Latin American biomedical community considers the PubMed and SciELO as the best databases to search for journal articles, however, other areas of knowledge, have used other search sources, especially Google Scholar (GA). Objectives: To determine whether the GA can be a useful tool in search of theme referenced in scientific articles in medical education, compared to PubMed and SciELO. Methodology: This paper is a descriptive and documentary study with qualitative and quantitative analysis of scientific articles published in GA, PubMed and SciELO. In each search site it was used the following key words in English for data recovery: "medical curriculum" and "ethics". Results: After application of data mining technique in search platforms, 434 articles were obtained in GA, and 88 articles available on the website of the journal, 201 on site of the databases, 143 on other sites and just two in blogs. On PubMed 10 scientific articles were obtained, with 8 available in the database and two in periodic site. Finally, on SciELO found only one article, which was available in their own database. Qualitatively, 382 relevant articles were found in GA, in PubMed and SciELO. Conclusion: The GA proved to be more efficient, both qualitatively and quantitatively, in rescuing scientific articles in medical education in the chosen theme.
\end{abstract}

\section{Keywords}

Bibliographic databases; Medical education; PubMed.

\section{Como você deve citar?}

PUCCINI, Lucas Rebelo Silva et al. Comparativo entre as bases de dados PubMed, SciELO e Google Acadêmico com o foco na temática Educação Médica. Cadernos UniFOA, Volta Redonda, n. 28, p. 75-82, ago. 2015. 


\section{INTRODUÇÃO}

Antes da era da internet, o acesso a um determinado artigo científico era mais difícil e complexo. Em alguns casos, tornava-se quase inacessível aos pesquisadores, devido à dificuldade do acesso ao banco de dados para a localização do artigo ou ao custo da obtenção do mesmo. Atualmente, com a crescente disponibilização de publicações científicas em formato eletrônico na web e a criação de redes de pesquisadores e bancos de dados, disponibilizando essas publicações, o acesso começa a ser universal (MUELLER, 1999; FALAGAS et al., 2008; CAREGNATO, 2011).

A busca e seleção de artigos científicos relevantes é uma habilidade que deve ser desenvolvida pelos médicos que necessitam de constante atualização. A comunidade biomédica latino-americana, em geral, utiliza-se do Medline, parte integrante do PubMed (http://www.ncbi.nlm.nih.gov/pubmed) e da SciELO (http://www.scielo.org), como bases de dados informatizadas de acesso gratuito para pesquisa de artigos científicos, fontes primárias de informação (BERNARDO, 2004). Em outras áreas de conhecimento, como em ciências sociais e humanidades, entretanto, não há bancos tão extensos ou abrangentes (CAREGNATO, 2011). Nessas áreas, outras fontes de busca têm sido utilizadas, sendo que o Google Acadêmico (GA) tem se projetado como um dos principais, uma vez que o resgate de artigos científicos no GA é feito por toda a web, teoricamente não possuindo limites (FALAGAS et al., 2008). Outra questão é que o Google é muito utilizado pelos usuários, devido à sua simplicidade, rapidez e amplitude de informação (GIUSTINI et al., 2005).

O que se questiona, entretanto, é a quantidade e a qualidade do que se resgata pelo GA comparadas aos bancos de dados amplamente aceitos pelos cientistas. A qualificação dos periódicos é uma importante ferramenta para avaliar a credibilidade dos artigos científicos publicados nas respectivas bases de dados. Em nível nacional, essa avaliação é feita baseada na classificação de periódicos da Coordenação de Aperfeiçoamento de Pessoal de Nível Superior: Qualis/CAPES. A posição do periódico na escala Qualis depende do fator de impacto - FI (ISI/SCOPUS) (CAPES). O Fl é publicado anualmente pelo Institute of Scientific Information -ISI da Thomson Scientific, que existe desde 1960 e é utilizado como índice para avaliar o impacto das publicações de um determinado periódico em escala internacional. 0 cálculo do $\mathrm{Fl}$ é baseado na quantidade de artigos científicos publicados no periódico, no período de dois anos (QP) e quantas vezes os artigos desse periódico foram citados em outras publicações, nos últimos dois anos (QC). A fórmula é dada pela divisão de $\mathrm{QC}$ por $\mathrm{QP}$ e, pelo resultado disso, é gerado a pontuação do $\mathrm{Fl}$.

Uma das áreas de interesse no meio médico é a Educação Médica. Essa área vem expandindo continuamente em outros países, inclusive no Brasil, com um número crescente de revistas exclusivas ou que dedicam espaço para o tema. Uma das questões a serem respondidas é se as temáticas em educação médica podem ser resgatadas nos mesmos bancos de dados utilizados para a pesquisa biomédica classicamente aceita ou se há lacunas. 0 GA poderia resgatar artigos de qualidade nessa temática?

\section{OBJETIVOS}

O objetivo geral do estudo visa verificar se o GA pode ser uma ferramenta de busca útil no resgate de artigos científicos referenciados na temática em educação médica, comparados ao PubMed e SciELO. De modo específico, tem-se o intuito de verificar comparativamente, em termos quantitativos e qualitativos, as publicações encontradas, verificar se a metodologia de busca interfere no resgate dos artigos científicos e qual a abrangência do resgate comparativo entre os bancos de dados definidos. 


\section{MÉTODOS}

O presente trabalho é um estudo descritivo e documental de natureza qualiquantitativa sobre artigos científicos completos publicados na Internet, no período entre 01 de janeiro de 2011 a 31 de dezembro de 2011, utilizando as bases de dados PubMed, GA e SciELO. Foram utilizadas as seguintes palavras-chave em inglês para a recuperação de dados: "medical curriculum" and "ethics". Após a busca das palavras-chave nas bases de dados, foram contabilizados os resultados em cada sítio. Os resultados foram submetidos à análise de dados, na qual foram excluídas teses (doutorado e mestrado), textos de livros, repetições, artigos científicos com acesso indisponível e citações de artigos, restando apenas os artigos científicos com acesso disponível. Foi analisada a procedência dos artigos científicos: sítios dos periódicos, sítios das bases de dados, blogs ou em outros sítios que podem ser de universidades, por exemplo. Em seguida, foi analisado se os artigos científicos completos eram de acesso livre (gratuito) ou restrito (pago).

Em termos quantitativos, foi verificado quantos artigos científicos fazem parte, simultaneamente, das coleções do GA, PubMed e SciELO. Não houve restrição da língua na seleção de artigos.

A qualidade dos artigos científicos foi verificada através da pertinência do resumo do artigo, em relação aos assuntos currículo médico e ética. Foi avaliado também o $\mathrm{Fl}$ e/ou Qualis dos periódicos da revista, na qual estava inserido o artigo.

Apresenta-se um fluxograma (Figura 1) que sintetiza o método utilizado na pesquisa.

Figura 1 - Diagrama do método utilizado no estudo.

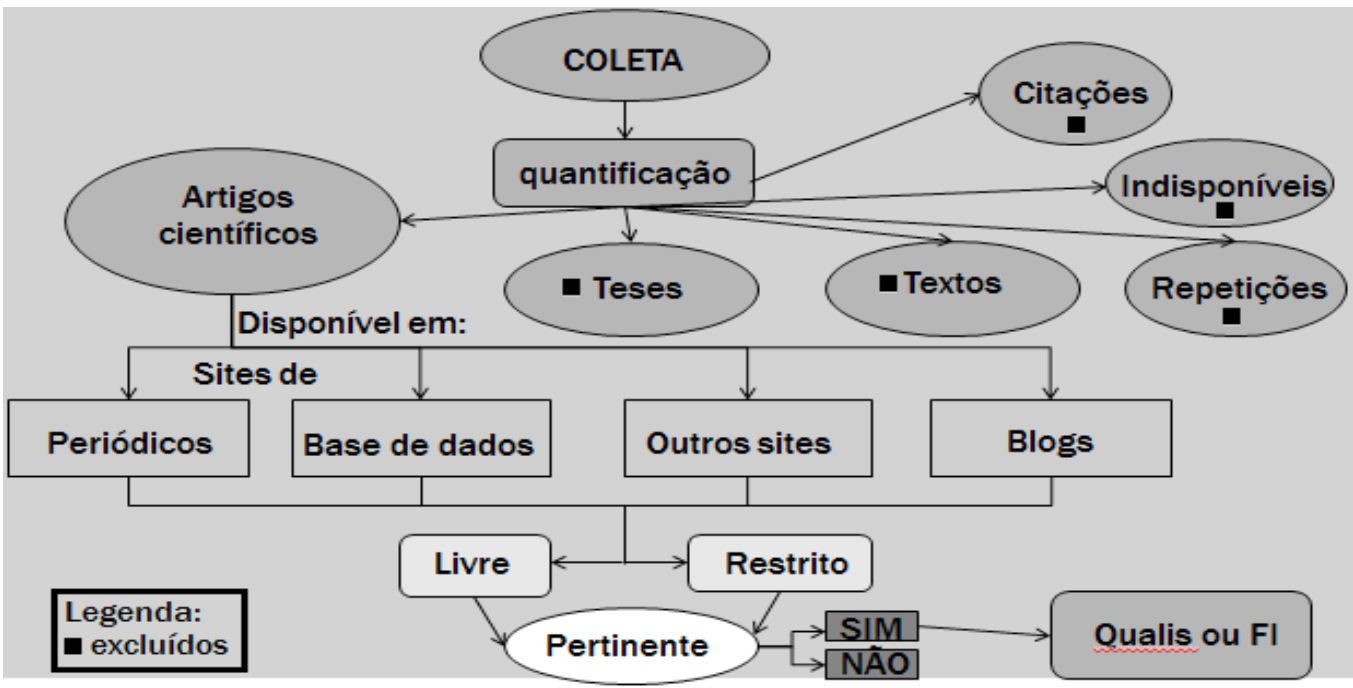

Fonte: dos autores.

\section{RESULTADOS}

A partir da aplicação do método nas bases de dados, foram obtidos 434 artigos científicos no GA, 10, no PubMed e; apenas um, no SciELO. 
Apesar de a busca no PubMed resgatar 10 artigos com os descritores citados, verificou-se que, na busca no GA, mais quatro artigos do PubMed foram localizados e que não havia sido resgatados primariamente pela mesma. O mesmo ocorreu com a base SciELO que, apesar de ter como resultado na busca apenas um artigo, no GA, foram resgatados mais 11 artigos com os mesmos descritores, todos indexados na base SciELO. Foram resgatados seis artigos científicos concomitantemente pelo GA e PubMed.

Dos 434 artigos científicos encontrados no GA, 88 estavam em sítios eletrônicos dos próprios periódicos, dentre os quais: Medical Teacher, Medical Education, British Medical Journal Quality and Safety, Plos One e BMC Medical Education. Em sítios de bases de dados como NCBI, SciELO, Online Library Wiley, Elservier e Springerlink, foram encontrados 201 artigos científicos. Cento e quarenta e três artigos foram localizados em outros sítios eletrônicos pertencentes a universidades como Oxford, Cambridge, University of California, Manipal, Aberdeen, Queensland e dois, em blogs.

Em relação aos artigos encontrados no PubMed, oito estavam disponíveis no sítio da base do próprio Pubmed e dois, em sítios de periódicos. Por fim, o único artigo encontrado pelo SciELO, estava disponível em sua própria base de dados. A distribuição dos artigos encontrados está no Quadro 1.

Quadro 1 - Localização na web dos artigos científicos.

\begin{tabular}{|l|c|c|c|}
\hline & GA & PubMed & SciELO \\
\hline Artigos disponíveis no sítio dos periódicos & 88 & 2 & 0 \\
\hline Artigos disponíveis no sítio das bases de dados & 201 & 8 & 1 \\
\hline Artigos disponíveis em sítio & 143 & 0 & 0 \\
\hline Artigos disponíveis em blogs & 2 & 0 & 0 \\
\hline Total & 434 & 10 & 1 \\
\hline
\end{tabular}

Fonte: dos autores.

Em relação ao acesso dos artigos completos, disposto no Quadro 2, pelo GA, foram resgatados 321 artigos de acesso livre e 113, de acesso restrito. Pelo PubMed, apenas três artigos foram de acesso livre e sete, de acesso restrito. Quanto ao SciELO, essa biblioteca eletrônica tem uma política de acesso a todos textos completos de artigos, portanto, todos os artigos resgatados são de acesso livre.

Quadro 2 - Acesso dos artigos científicos

\begin{tabular}{|l|c|c|c|}
\hline & GA & PubMed & SciELO \\
\hline Artigos com o acesso restrito & 113 & 7 & 0 \\
\hline Artigos com o acesso aberto & 321 & 3 & 1 \\
\hline Total & 434 & 10 & 1 \\
\hline
\end{tabular}

Fonte: dos autores.

Sobre a pertinência dos artigos em relação ao tema escolhido, dos 434 artigos encontrados pelo GA, 282 (65\%) foram considerados pertinentes. Todos os artigos encontrados pelo PubMed e SciELO foram pertinentes à temática proposta. 
Do ponto de vista da qualidade dos periódicos em que se encontravam publicados os artigos, verificou-se que, em relação ao GA, os 282 artigos pertinentes encontrados pertenciam a um total de 90 periódicos. Desse total, 42 periódicos eram indexados ao ISI, com fator de impacto variando de 0,12 a 30,026, o que significou que, do total de artigos resgatados, somente 191 artigos pertenciam a essas revistas. Em relação ao Qualis/CAPES da publicação, 177 artigos pertenciam aos periódicos com classificação variando de B5 a A1, todos na área de avaliação em Medicina I.

Em relação ao PubMed, oito dos 10 artigos pertenciam a periódicos qualificados por fator de impacto e, no SciELO, o único resgatado também pertencia a um periódico com Fl. Esses mesmos periódicos também estavam classificados no Qualis/CAPES.

O número de periódicos encontrados distribuídos pela classificação Qualis/CAPES e pelo Fator de Impacto para cada banco analisado está disposto no Quadro 3.

Quadro 3 - Distribuição do Qualis/CAPES e FI dos periódicos resgatados nas bases de dados estudadas.

\begin{tabular}{|l|c|c|c|c|}
\hline \multicolumn{5}{|c}{ Qualificação dos periódicos dos artigos científicos } \\
\hline Qualis/CAPES & FI & PubMed & SciELO & GA n (\%) \\
\hline A1 & 3,800 ou mais & 1 & 0 & $13(4,6)$ \\
\hline A2 & 2,500 a 3,799 & 0 & 0 & $42(14,9)$ \\
\hline B1 & 1,300 a 2,499 & 0 & 0 & $46(16,3)$ \\
\hline B2 & 0,001 a 1,299 & 7 & 1 & $92(32,6)$ \\
\hline B3 & não possui & 0 & 0 & $26(9,2)$ \\
\hline B4 & não possui & 0 & 0 & $9(3,2)$ \\
\hline B5 & não possui & 0 & 0 & $11(3,9)$ \\
\hline Não possui & não possui & 2 & 0 & $43(15,3)$ \\
\hline TOTAL & & 10 & 1 & $282(100)$ \\
\hline
\end{tabular}

Fonte: dos autores.

\section{DISCUSSÃO}

No campo biomédico, a pioneira no desenvolvimento de ferramenta de busca acessível e interativa foi a National Library of Medicine (NLM) que, em 1971, criou o Medline. Posteriormente, houve, a partir da fusão do Old Medline, em 1996, com o Medline, a criação do PubMed, que tornou-se a maior fonte de referências de artigos científicos no meio biomédico (FALAGAS et al., 2008). O Old Medline continha citações e revistas adicionais, incluindo cerca de dois milhões de citações de artigos publicados entre 1950 e 1965. O Medline possui uma abrangência de cerca de 4.800 títulos de revistas publicadas nos Estados Unidos e em 70 outros países (STEINBROOK, 2006). A função do PubMed (http://www.ncbi. nlm.nih.gov/pubmed) é ser um arquivo digital e livre, que visa à preservação da informação científica em Ciências da Saúde. Seu objetivo é reunir periódicos tradicionais como o BMJ - British Medical Journal e o Lancet, por exemplo, com os novos periódicos, em formato digital, com bases de dados bibliográficos, como a Medline e outros produtos de informação científica em Ciências da Saúde, produzida nos 
Estados Unidos (NORONHA, 2011). O PubMed, por ser muito útil, rápido e livre, justifica a autoridade que ganhou ao longo dos anos (FALAGAS et al., 2008).

Outra base de dados disponível para pesquisa, importante no meio latino-americano em saúde, é a biblioteca digital SciELO, que surgiu por meio da associação entre a Fundação de Amparo à Pesquisa de São Paulo (FAPESP) e a base de dados Bireme. Em 2002, o projeto SciELO ganhou o apoio do Conselho Nacional de Desenvolvimento Científico e Tecnológico (CNPq). O SciELO abrange periódicos de todo território latino-americano e Caribe, especializados em ciências da saúde, entre outras áreas do conhecimento. A base de dados SciELO, atualmente, pode ser recuperada por diferentes ferramentas de busca não restritas ao portal SciELO, como o GA (http://scholar.google.com), o PubMed, a Biblioteca Virtual em saúde (BVS) e o Portal de Periódicos Capes, entre outros. Em 2008, o SciELO registrou uma média mensal de oito milhões de acessos a textos completos de artigos científicos, sendo que cerca da metade foi por meio do GA (ABDALA et al., 2008-2009).

Criado em novembro de 2004, na versão em língua inglesa, o GA foi disponibilizado em português em janeiro de 2006. Ele fornece uma maneira simples de pesquisar literatura acadêmica de forma abrangente e é útil para pesquisar várias disciplinas e fontes em apenas um lugar: artigos revisados por especialistas, teses, livros, resumos e artigos de editoras acadêmicas, organizações profissionais, bibliotecas de pré-publicações, universidades e outras entidades acadêmicas (GOOGLE ACADÊMICO). Com todas suas vantagens substanciais, por ser um motor de busca com caixa de consulta simples e com a facilidade no uso, o GA não deixa de ser isento de falhas. Há críticas em relação suas funções de pesquisa avançada, que pode não ser confiável ou atual, por não possuir limites do seu alcance e cobertura, devido à falta de recursos e opções para classificação e limitação do seu conteúdo (SHULTZ, 2007). Sobre a sua cobertura garantida, é uma questão de grande importância no meio científico, quando é necessária uma ampla pesquisa bibliográfica para a realização de ensaios clínicos, revisões sistemáticas, entre outros estudos (GIUSTINI et al., 2005) Já a abrangência em outras bases de dados como PubMed e Web of Science, são mais confiáveis e, portanto, mais garantidas quando se tem tais objetivos científicos (MUGNAINI et al., 2008).

Com base no conhecimento da abrangência de cada base de dados como plataforma de busca, observa-se que, dentre as citadas, a que possuiu uma maior abrangência para a busca de artigos científicos foi o GA. Seu alcance é por toda a Web (teoricamente sem limites), encontrando, inclusive, artigos científicos do PubMed, que não haviam sido resgatados no próprio PubMed. O mesmo ocorreu com os artigos do Scielo, que, na busca na própria biblioteca virtual, só resgatou um artigo, enquanto que o GA resgatou 12 artigos indexados na base SciELO.

Apesar da imensa capacidade de resgate de artigos o GA apresentou (434), há também uma grande quantidade de artigos que não corresponde ao tema proposto. Aproximadamente $35 \%$ dos artigos encontrados não foram pertinentes ao tema "medical curriculum and ethics". Isso demonstra que deve ser realizada uma avaliação mais minuciosa dos resultados encontrados no GA. Quanto ao PubMed e ao SciELO, a desvantagem foi a de resgatar um número bem menor de artigos científicos em relação ao GA. Os artigos resgatados por esses bancos, entretanto, apresentavam pertinência nesse assunto, sugerindo uma maior fidedignidade no assunto buscado, quando se opta por essas bases. A diferença do número de artigos resgatados parece estar relacionada ao modo de busca utilizada por cada banco ou pela natureza dos periódicos indexados. O PubMed apresenta um foco biomédico, com ênfase nas ciências naturais e na saúde, sendo possível que revistas voltadas à educação, mesmo que na área médica, não estejam contempladas. Em relação ao SciELO, a justificativa parece ser pela metodologia de busca de palavras, que, no GA, é realizada por todo corpo do título e do texto e, no SciELO, apenas por meio do uso de descritores específicos, como palavras-chave. 
Existem também outras bases de dados de grande importância como o Scopus e o Web of Science, que são abrangentes com artigos em diversas áreas de conhecimento. O Scopus, uma base europeia pertencente à editora Elsevier, possui uma vasta coleção, com, aproximadamente, 12.850 periódicos (FALAGAS et al., 2008). Já o Web of Science, pertencente ao Institute of Scientific Information (ISI) dos Estados Unidos, possui cerca de 8700 periódicos e disponibiliza análises de citações, oferecendo bons recursos gráficos. As duas bases de dados, por apresentarem acesso restrito e não serem gratuitas, como o PubMed, o SciELO e o GA, não são, em geral, as bases mais consultadas pela comunidade biomédica (FALAGAS et al., 2008).

No PubMed e no GA, apesar de estarem disponíveis para a consulta gratuitamente e apresentam vários artigos apenas com acesso gratuito a resumos, oferece textos completos apenas na modalidade paga, ao contrário da biblioteca SciELO, que disponibiliza todos os artigos em textos completos gratuitamente. No presente trabalho, verificou-se que, do total de artigos resgatados pelo GA, $26 \%$ necessitavam de pagamento para o acesso aos textos completos. Em relação aos artigos resgatados no PubMed, $70 \%$ são de acesso restrito, com necessidade de pagamento. Uma das vantagens, portanto, da utilização do GA em relação ao PubMed pode ser a sua maior acessibilidade e democratização da informação.

O FI tem importância para bibliotecários na seleção de periódicos para as coleções da biblioteca, e, em alguns países, é utilizado para avaliar os pesquisadores e instituições, para efeitos de promoção acadêmica e alocação de recursos. Há opiniões contrárias a seu respeito, quanto à fidedignidade de avaliar a qualidade de um periódico, pois os próprios pesquisadores não votam na qualificação das pesquisas. Todavia, é a ferramenta em vigência mais utilizada e aceita para tal avaliação (SAHA et al., 2003). Em suma, as características indispensáveis para que um periódico alcance um status considerável no meio científico é a competitividade, o impacto e a visibilidade internacional (FORATTINI, 1996). Com isso, demonstra a intensa busca dos periódicos em publicar mais artigos de boa qualidade e serem cada vez mais citados, para alcançarem um FI de mais prestígio e, consequentemente, mais confiáveis cientificamente. Concomitantemente, essas bases de dados para pesquisa, ao resgatarem mais artigos científicos de periódicos bem cotados no $\mathrm{Fl}$, alcançam também um prestígio maior para o resgate de conhecimento científico.

A partir da análise dos resultados do $\mathrm{GA}, 65 \%$ dos artigos científicos foram considerados pertinentes e destes, no quesito $\mathrm{Fl}, 68,4 \%$ dos periódicos estão contemplados. No PubMed, $80 \%$ possuem $\mathrm{FI}$ e, no SciELO, todos (o único). Cerca de $20 \%$ dos artigos científicos pertinentes encontrados no GA estão em periódicos com FI maior que 2,500. Verifica-se, portanto, que é possível resgatar, não só um maior número de artigos publicados no $\mathrm{GA}$, quando comparado às outras duas bases de dados, como também artigos de qualidade, segundo as classificações do FI e Qualis/CAPES.

\section{CONCLUSÃO}

Com base nos resultados obtidos no presente estudo, concluiu-se que o GA demonstrou maior eficiência em resgatar artigos científicos qualiquantitativamente. Embora o PubMed seja considerado a melhor plataforma de busca na área biomédica e o SciELO difundido no cenário Latino-americano, o GA mostrou-se muito útil na busca de artigos referenciados na temática estudada.

O Google Acadêmico foi projetado como ferramenta para recuperação da informação para qualquer tipo de público e pode ser útil para algumas disciplinas em especial (Caregnato, 2011; Harzing, 2014; Winter et al., 2014). Deve-se observar, entretanto, como salientam alguns autores, que essa ferramenta não deve ser utilizada para avaliar o impacto das publicações ou indicadores de produção científica (Jacsó, 2010). Outra observação é que, apesar de um maior resgate de artigos científicos, a pertinência ao assunto procurado e a qualidade dessa publicação devem ser avaliadas com mais cautela. 


\section{REFERÊNCIAS}

ABDALA, Carmen Verônica Mendes; ANDRADE, Vinícius Antônio. Recuperação de informações baseada em clusters. REVISTA USP, São Paulo, n.80, p. 54-61, dezembro/fevereiro 2008-2009.

BERNARDO, Wanderley Marques; et al. A prática clínica baseada em evidências . parte II - Buscando as evidências em fontes de informação. Rev Assoc Med Bras 2004; 50(1): 104-8.

CAPES. Disponível em: <http://www.capes.gov.br/avaliacao/qualis>. Acesso em: 07 mar. 2013.

CAREGNATO, Sonia Elisa. Google Acadêmico como ferramenta para os estudos de citações: Avaliação da Precisão das Buscas por Autor. Ponto de Acesso, Salvador, V.5, n.3 p. 72-86 dez 2011.

FALAGAS, Matthew E; PITSOUNI, Eleni I.; MALIETZIS, George A.; PAPPAS, Georgious. Comparison of PubMed, Scopus, Web of Science, and Google Scholar: strengths and weaknesses. Scientific Databases, Pros and cons. The FASEB Journal, Vol. 22 February 2008.

FORATTINI, O.P. A tríade da publicação científıca. Revista de Saúde Pública, São Paulo, v. 30, n. 1, 1996.

GIUSTINI D, BARSKY E: "A look at Google Scholar, PubMed, and Scirus: comparisons and recommendations". Journal of the Canadian Health Libraries Association, 26(3):85-9, 2005.

GOOGLE ACADÊMICO. Disponível em: <http://scholar.google.com.br/intl/pt-BR/scholar/about.html>. Acesso em: 07 jun. 2013.

HAMAMOTO FILHO, Pedro Tadao; et al. Pesquisa em Educação Médica Conduzida por Estudantes: um Ano de Experiência do Núcleo Acadêmico de Pesquisa em Educação Médica. Rev. Bras. de Educação Médica. 35 (1): 108-113; 2011.

HARZING, Anne-Wil. A longitudinal study of Google Scholar coverage between 2012 and 2013. Scientometrics 98:565-575, 2014.

JACSÓ, Péter. Metadata mega mess in Google Scholar. Emerald Group Publishing Limited, Online Information Review. Vol. 34, $\mathrm{n}^{\circ} 1,2010$.

MUELLER, Suzana Pinheiro Machado. O círculo vicioso que prende os periódicos nacionais. Datagramazero, 1999.

NORONHA, Ilma Maria Worsth. O livre acesso à informação científica em doenças negligenciadas: um estudo exploratório. Mestrado em ciência da informação. Universidade Federal Fluminense - UFF, Instituto de arte e comunicação social. Programa de pós-graduação em ciência da informação. Niterói, 2011.

SAHA, Somnath; et al. Impact factor: a valid measure of journal quality? J Med Libr Assoc. 91(1). Jan. 2003.

SHULTZ M. Comparing test searches in PubMed and Google Scholar. J Med Libr Assoc; 95(4):442-445, 2007.

STEINBROOK R. Searching for the right search: reaching the medical literature (editorial). N Engl J Med; 354(1):4-7, 2006.

WINTER, Joost C. F.; ZADPOOR, Amir A.; DODOU, Dimitra. The expansion of Google Scholar versus Web of Science: a longitudinal study. Scientometrics. 98:1547-1565, 2014 